\title{
Enhancement factors for grounded ice and ice shelves inferred from an anisotropic ice-flow model
}

\author{
Ying MA, ${ }^{1}$ Olivier GAGLIARDINI, ${ }^{1}$ Catherine RITZ, ${ }^{1}$ Fabien GILLET-CHAULET, ${ }^{2}$ \\ Gaël DURAND, ${ }^{1}$ Maurine MONTAGNAT ${ }^{1}$ \\ ${ }^{1}$ Laboratoire de Glaciologie et Géophysie de I'Environnement, CNRS/Université Joseph Fourier - Grenoble I, \\ 54 rue Molière, BP 96, 38402 Saint-Martin-d'Hères Cedex, France \\ E-mail: gagliar@Igge.obs.ujf-grenoble.fr \\ ${ }^{2}$ British Antarctic Survey, Natural Environment Research Council, Madingley Road, Cambridge CB3 OET, UK
}

\begin{abstract}
Polar ice is known to be one of the most anisotropic natural materials. For a given fabric the polycrystal viscous response is strongly dependent on the actual state of stress and strain rate. Within an ice sheet, grounded-ice parts and ice shelves have completely different stress regimes, so one should expect completely different impacts of ice anisotropy on the flow. The aim of this work is to quantify, through the concept of enhancement factors, the influence of ice anisotropy on the flow of grounded ice and ice shelves. For this purpose, a full-Stokes anisotropic marine ice-sheet flowline model is used to compare isotropic and anisotropic diagnostic velocity fields on a fixed geometry. From these full-Stokes results, we propose a definition of enhancement factors for grounded ice and ice shelves, coherent with the asymptotic models used for these regions. We then estimate realistic values for the enhancement factors induced by ice anisotropy for grounded ice and ice shelves.
\end{abstract}

\section{INTRODUCTION}

The crystallographic structure of an ice crystal presents a hexagonal symmetry, resulting in a strong visco-plastic anisotropy. An ice crystal deforms mainly due to sliding in its basal planes, which are the planes perpendicular to its hexagonal symmetry axis (i.e. its $c$-axis). Due to the deformation, the $c$-axes of the ice crystals turn to preferential orientations depending on the strain-rate and stress history, leading to different types of fabric. For example, under polar-ice-sheet conditions in uniaxial compression the $c$ axes rotate towards the compressional axis, leading to singlemaximum fabrics, whereas in uniaxial tension the $c$-axes rotate towards a plane perpendicular to the tension axis, leading to girdle-type fabrics (Van der Veen and Whillans, 1994). Due to the great variety of flow conditions prevailing in ice sheets, the type of fabric varies greatly from place to place.

As a consequence of its strong crystal anisotropy, polycrystalline ice is one of the most anisotropic natural materials, but its anisotropy is strongly related to the distribution of the crystal orientations. If ice crystals in a polycrystal are distributed randomly, the mechanical behaviour of the polycrystal is isotropic. Moreover, for the same given fabric, the response of the polycrystal is strongly dependent on the state of stress or strain rate. For example, as observed experimentally by Pimienta and others (1987), it is 25 times easier to deform a polycrystal with a singlemaximum fabric in shear perpendicular to the mean $c$-axis orientation than in uniaxial compression along the same orientation.

The grounded parts of ice sheets are dominated by vertical compression near domes and near the ice surfaces and by horizontal shear elsewhere. Under a dome most of the observed fabrics are almost axisymmetric and approximately centred around the in situ vertical axis. They evolve from isotropy at the surface to single-maximum fabrics near the bedrock (Thorsteinsson and others, 1997; Durand and others, 2009a). Along the flanks the vertical evolution of the fabric is more complex and only a few deep cores drilled along flanks have been studied. It seems that for such locations the fabric evolves first toward girdle shapes in the upper part of the core, and then to a single-maximum fabric deeper, where vertical compression and shear stresses dominate (Eisen and others, 2007; Seddik and others, 2008). In both cases, these types of fabric are easier to shear than isotropic ice, and the anisotropy induces an enhancement of the flow compared with an isotropic reference (Mangeney and others, 1996).

In the absence of basal friction, ice shelves are dominated by longitudinal stretching and lateral shearing. Only a few authors have studied ice-shelf fabrics (Kirchner and Faria, 2009; Treverrow, 2009). As shown by Craven and others (2005, 2009), the Amery Ice Shelf, East Antarctica, is structured in three layers. The first thin layer near the surface is composed of meteoritic ice formed by compaction of the snow accumulated on the ice shelf itself. Deeper, a thick layer contains the ice which has flowed from the continent. Its fabric is thus inherited from its initial fabric when entering the ice shelf and the stress and strain rate undergone within its travel through the ice shelf. At the bottom, a third layer, which can be up to $200 \mathrm{~m}$ thick, is formed by the accretion of marine ice. The distribution of marine ice under ice shelves is not uniform, due to ocean circulation (Fricker and others, 2001). Physically, marine ice is heavier than meteoritic ice and it is permeable and isothermal due to the presence of sea water in the pores (Craven and others, 2009). In borehole studies of the Amery Ice Shelf, Treverrow (2009) observed girdletype fabrics and also multiple-maxima crystal orientation fabrics. These latter fabrics are characteristic of migration recrystallization processes which can be initiated by high enough temperatures and/or stresses, whereas a girdle shape is characteristic of strain-induced fabrics in tension. When considering only strain-induced fabrics, polycrystal models indicate that for a tension state of stress, ice becomes more and more stiff, because of the fabric evolution to a more 
textured girdle-type fabric (Castelnau and others, 1996). As tension dominates the state of stress in ice shelves, we expect the ice in ice shelves to be stiffer than an isotropic reference, due to the strain-induced anisotropy.

In this paper, we compare isotropic and anisotropic velocity fields computed from a flowline model on a fixed geometry to estimate the flow enhancement induced solely by the ice anisotropy for both the grounded and the floating parts of an ice sheet. We first present the nonlinear full-Stokes anisotropic ice-flow model used for this study and define the enhancement factors for the grounded and floating part, respectively. These enhancement factors are then calculated for various flow configurations, by varying the basal conditions or the fabric field.

\section{CONTEXT OF ISOTROPIC ICE-SHEET MODELS}

In large-scale ice-sheet models, ice rheology is described using Glen's isotropic flow law, which links the deviatoric stress, $\boldsymbol{\tau}$, and the strain rate, $\dot{\varepsilon}$ :

$$
\dot{\varepsilon}=A \tau_{\mathrm{e}}^{n-1} \tau,
$$

where $\tau_{\mathrm{e}}^{2}=\tau_{i j} \tau_{i j} / 2$ is the square of the second invariant of the deviatoric stress and $A$ is a rheological parameter, which depends on the ice temperature via an Arrhenius law.

Another characteristic of large-scale ice-sheet models is that they solve simplified equations based on asymptotic expansions of the Stokes equations. These equations are obtained by retaining only the largest components of the deviatoric stress. For the grounded part, the dominant stress is the horizontal shear stress and the resulting set of equations is known as the shallow-ice approximation (SIA; Hutter, 1983). For ice shelves, the stress is dominated by the longitudinal component and this leads to the shallow-shelf approximation (SSA; MacAyeal, 1989). Classically, the SIA is solved for the grounded part and the SSA is used for ice shelves and the fast-flowing outlet glaciers. In some recent applications, both approximations are solved together on the whole ice sheet, so the SSA is used to determine the sliding velocity on the grounded part (Bueler and Brown, 2009).

When using Glen's flow law (Equation (1)), ice-sheet models show inconsistencies with the real characteristics of the ice flow, in an opposite way for grounded and iceshelf parts. In the grounded part the observed velocities are underestimated by the models and they are overestimated on ice shelves. To better reproduce realistic characteristics of ice sheets, many authors use an ad hoc coefficient to proportionately modify Glen's flow law to adjust their results. This empirical coefficient, $E$, called the enhancement factor, modifies Glen's flow law as follows:

$$
\dot{\varepsilon}=E A \tau_{\mathrm{e}}^{n-1} \boldsymbol{\tau} \text {. }
$$

For grounded ice, Mangeney and Califano (1998) developed the SIA equations for an anisotropic rheology restricted to transverse isotropy with a vertical rotational symmetry axis everywhere. They showed that, in the particular case of a linear rheology and a flat bedrock, the zero-order anisotropic SIA is equivalent to the use of an enhancement factor in the isotropic SIA. Further developments by Philip and Meyssonnier (1999) showed that, in the case of a non-vertical material symmetry axis, the diagonal components of the deviatoric stress can be of the same order of magnitude as the shear stress. These last developments indicate that the above conclusion of
Mangeney and Califano (1998) is only valid in the restricted case of a vertical material symmetry axis. For a grounded ice-flow regime, all the applications indicate that anisotropic ice flows faster than isotropic ice (Mangeney and Califano, 1998; Gagliardini and Meyssonnier, 1999; Staroszczyk and Morland, 2000), with an enhancement of the flow depending on the anisotropic polycrystal models. To our knowledge, the comparison of isotropic and anisotropic flows for an ice shelf has never been addressed, so the effect of anisotropy on ice-shelf flows remains unclear. Including a tensorial anisotropic flow law properly in a large-scale ice-sheet model is not straightforward, which explains why all existing ice-sheet models account for anisotropy effects using the concept of enhancement factors. A valuable alternative for ice-sheet models is the easy-to-implement CAFFE model (Continuum-mechanical Anisotropic Flow model based on an anistropic Flow Enhancement factor e.g. Placidi and others, 2010), which is based on an anisotropic flow enhancement factor and therefore preserves the scalar-viscosity form of Glen's law.

In some large-scale ice-sheet models, two different values of the enhancement factor are used, depending on the stress regime (e.g. Huybrechts, 1990). For grounded ice, an enhancement factor for shear stress, $E_{S I A}(>1)$, is introduced in the ice rheology for the SIA equations, whereas for ice shelves and ice streams an enhancement factor in tension, $E_{S S A}(<1)$, modifies Glen's law in the SSA equations. This contrast of rheology between the grounded ice and the ice shelf can be characterized by the ratio of the enhancement factor for the grounded part over the enhancement factor for ice shelves, i.e. $E_{S I A} / E_{S S A}$. In his model, Huybrechts (1990) used a contrast ratio of $E_{S I A} / E_{S S A}=5$. Ritz and Pollard (personal communications, 2009) apply a contrast ratio of 10 in their models. Although all the values of the contrast ratio used by these authors have the same trends, they were estimated empirically in order to obtain realistic results with the various ice-sheet models. The aim of this paper is to quantify which part of these enhancement factors can be attributed to strain-induced anistotropy, by comparing isotropic and anisotropic ice-flow results.

\section{THE ANISOTROPIC ICE-FLOW MODEL Anisotropic law and fabric evolution}

In this section, we briefly present the anisotropic ice-flow model used in this study. For more details the reader can refer to previous publications related to the model itself (GilletChaulet and others, 2005, 2006; Durand and others, 2007; Martín and others, 2009). The fabric is described using the concept of orientation tensors. The second- and fourth-order orientation tensors are defined as

$$
a_{i j}^{(2)}=\left\langle c_{i} c_{j}\right\rangle \text { and } a_{i j k l}^{(4)}=\left\langle c_{i} c_{j} c_{k} c_{l}\right\rangle,
$$

where $\boldsymbol{c}$ is the $\boldsymbol{c}$-axis unit vector and \langle\rangle denotes the average over all the grains that make up the polycrystal. In this continuum description of the fabric, the polycrystal represents the local behaviour of a representative elementary ice volume. By assuming that the fourth-order orientation tensor, $\mathbf{a}^{(4)}$, is given as a tensorial function of $\mathbf{a}^{(2)}$ (GilletChaulet and others, 2006), the fabric can be described in a very condensed way using only $\mathbf{a}^{(2)}$. By definition, $\operatorname{tr} \mathbf{a}^{(2)}=1$, so that only the first two eigenvalues, $a_{1}^{(2)}$ and $a_{2}^{(2)}$, and three Euler angles are needed to completely define the fabric. As 
a consequence, modelled fabrics are orthotropic, i.e. the $C$-axis distribution presents three orthogonal symmetry planes. Although the orthotropy is a simple form of the most general anisotropy, it is thought to be a good compromise between physical adequateness and simplicity. The secondorder orientation tensor allows us to describe all the observed fabric patterns: for random $c$-axis distributions the diagonal entries of $\boldsymbol{a}^{(2)}$ are $a_{11}^{(2)}=a_{22}^{(2)}=a_{33}^{(2)}=1 / 3$, for a singlemaximum fabric with its maximum in the third direction, $a_{33}^{(2)}>1 / 3$ and $a_{11}^{(2)} \approx a_{22}^{(2)}<1 / 3$, and for a girdle-type fabric in the plane $\left(x_{1}, x_{2}\right), a_{33}^{(2)}<1 / 3$ and $a_{11}^{(2)} \approx a_{22}^{(2)}>1 / 3$. When the material symmetry axes are those of the general reference frame, as for the three particular previous fabrics, the nondiagonal entries of $\boldsymbol{a}^{(2)}$ are nulls.

The behaviour of the polycrystal is described by the general orthotropic linear flow law (GOLF; Gillet-Chaulet and others, 2005). In this law, ice is assumed to behave as a linearly viscous orthotropic material. In the present paper, following Pettit and others (2007) and Martín and others (2009), the GOLF law is extended to a nonlinear form by adding an invariant in the anisotropic linear law. The simple choice is either to add the second invariant of the strain rate (Martín and others, 2009) or the second invariant of the deviatoric stress (Pettit and others, 2007). No theoretical or experimental results are presently available that allow us to discard either of these two solutions, and other solutions based on anisotropic invariants of the deviatoric stress and/or the strain rate are also possible. Here the nonlinearity of the law is introduced through the second invariant of the deviatoric stress. With this definition, the anisotropy factors of the polycrystalline law for a given stress are identical in the linear and nonlinear cases. In other words, for a given fabric and a given state of stress, the corresponding strain rate relative to the isotropic response is the same for the linear and nonlinear cases. Using the strain-rate invariant, in the same way as Martín and others (2009), leads to different anisotropy factors (as defined here) in the linear and nonlinear cases. Therefore, the proposed expression of the nonlinear GOLF law is

$$
\begin{array}{r}
\sum_{r=1}^{3}\left[\eta_{r} \operatorname{tr}\left(\boldsymbol{M}_{r} \cdot \dot{\boldsymbol{\varepsilon}}\right) \boldsymbol{M}_{r}^{D}+\eta_{r+3}\left(\dot{\boldsymbol{\varepsilon}} \cdot \boldsymbol{M}_{r}\right.\right. \\
\left.\left.+\boldsymbol{M}_{r} \cdot \dot{\boldsymbol{\varepsilon}}\right)^{D}\right] \\
=2 A \tau_{\mathrm{e}}^{n-1} \boldsymbol{\tau},
\end{array}
$$

where $A$ is the temperature-dependent Glen's law parameter for isotropic ice. The six dimensionless anisotropy viscosities $\eta_{r}\left(\mathbf{a}^{(2)}\right)$ and $\eta_{r+3}\left(\mathbf{a}^{(2)}\right)(r=1,2,3)$ are functions of eigenvalues of the second-order orientation tensor, $\mathbf{a}^{(2)}$, which represent a measure of the anisotropy strength. The three structure tensors, $\mathbf{M}_{r}$, are given by the dyadic products of the three eigenvectors of $\mathbf{a}^{(2)}$, which then represent the material symmetry axes. In the method proposed by Gillet-Chaulet and others (2006), the six dimensionless viscosities, $\eta_{r}\left(\mathbf{a}^{(2)}\right)$, are tabulated as a function of the fabric strength (i.e. the $a_{i}^{(2)}$ ) using a micro/macro model. When the ice is isotropic, $\eta_{r}=0$ and $\eta_{r+3}=1(r=1,2,3)$, and Equation (4) reduces to Glen's flow law (Equation (1)).

Following Gillet-Chaulet and others (2006), the six dimensionless viscosities, $\eta_{r}\left(\mathbf{a}^{(2)}\right)$, are tabulated using the viscoplastic self-consistent (VPSC) model (Castelnau and others, 1996, 1998). The two crystal parameters in the VPSC model used to tabulate the GOLF law were chosen to reproduce the experimentally observed polycrystal anisotropy. Following
Gillet-Chaulet and others (2006), we use the shear strain rates ratio for a polycrystal with a single-maximum fabric and an isotropic polycrystal both experiencing the same shear stress. This anisotropy factor in shear is hereafter denoted $k_{\mathrm{s}}$ and, according to the experimental results of Pimienta and others (1987), $k_{\mathrm{s}} \approx 10$. In other words, the VPSC parameters are chosen so that under simple shear a polycrystal with a single-maximum fabric is $k_{\mathrm{s}}$ times easier to deform than the corresponding isotropic polycrystal. The experimental results of Pimienta and others (1987) indicate that an isotropic polycrystal is much easier to deform than a single-maximum fabric polycrystal experiencing the same uniaxial compressional stress. These experiments allow the definition of a second anisotropy factor for uniaxial compressional stress, denoted $k_{\mathrm{c}}$. In what follows, the anisotropic polycrystal response deduced from the GOLF law (Equation (4)) is such that $k_{\mathrm{s}}=10$ and $k_{\mathrm{c}}=0.4$. The value $k_{\mathrm{c}}=0.4$ is in accordance with the experimental results of Pimienta and others (1987) obtained from uniaxial compression tests on polycrystals with a single-maximum fabric. The anisotropy factors $k_{\mathrm{s}}$ and $k_{\mathrm{c}}$ are independent of Glen's flow-law exponent, $n$, with the adopted nonlinear formulation.

Assuming that recrystallization processes do not occur and that the ice fabric is induced solely by deformation, the evolution of the second-order orientation tensor, $\mathbf{a}^{(2)}$, can be written as

$$
\frac{\mathrm{D} \mathbf{a}^{(2)}}{\mathrm{D} t}=\boldsymbol{W} \cdot \mathbf{a}^{(2)}-\mathbf{a}^{(2)} \cdot \boldsymbol{W}-\left(\boldsymbol{C} \cdot \mathbf{a}^{(2)}+\mathbf{a}^{(2)} \cdot \boldsymbol{C}\right)+2 \mathbf{a}^{(4)}: \boldsymbol{C}
$$

where $\boldsymbol{W}$ is the spin tensor, defined as the antisymmetric part of the velocity gradient. The tensor $\boldsymbol{C}$ is defined as

$$
\boldsymbol{C}=(1-\alpha) \dot{\boldsymbol{\varepsilon}}+\alpha k_{\mathrm{s}} A \tau_{\mathrm{e}}^{n-1} \boldsymbol{\tau}
$$

The interaction parameter, $\alpha$, controls the relative weighting of the strain rate and the deviatoric stress in the fabricevolution equation (Equation (5)). When $\alpha=0$ the fabric evolution is solely controlled by the state of the strain rate, whereas when $\alpha=1$ the fabric evolves solely under the influence of the deviatoric stress. In between, as for the VPSC, both the strain rate and deviatoric stress contribute to the fabric evolution. In what follows, the interaction parameter is set to $\alpha=0.06$, in accordance with the crystal anisotropy and the VPSC model used to derive the polycrystal behaviour (Gillet-Chaulet and others, 2006). In Equation (5), the fourth-order orientation tensor is evaluated assuming a closure approximation giving $\mathbf{a}^{(4)}$ as a tensorial function of $\mathbf{a}^{(2)}$ (Gillet-Chaulet and others, 2006).

\section{Flow equations}

As shown in Figure 1, the domain geometry represents a two-dimensional flowline ice sheet and ice shelf. This geometry is the steady-state solution obtained with the same model assuming an isotropic rheology for step 12 of the MISMIP 3a experiment (Marine Ice Sheet Model Intercomparison Project; Durand and others, 2009b). Here we summarize the set-up of this experiment. The bedrock 


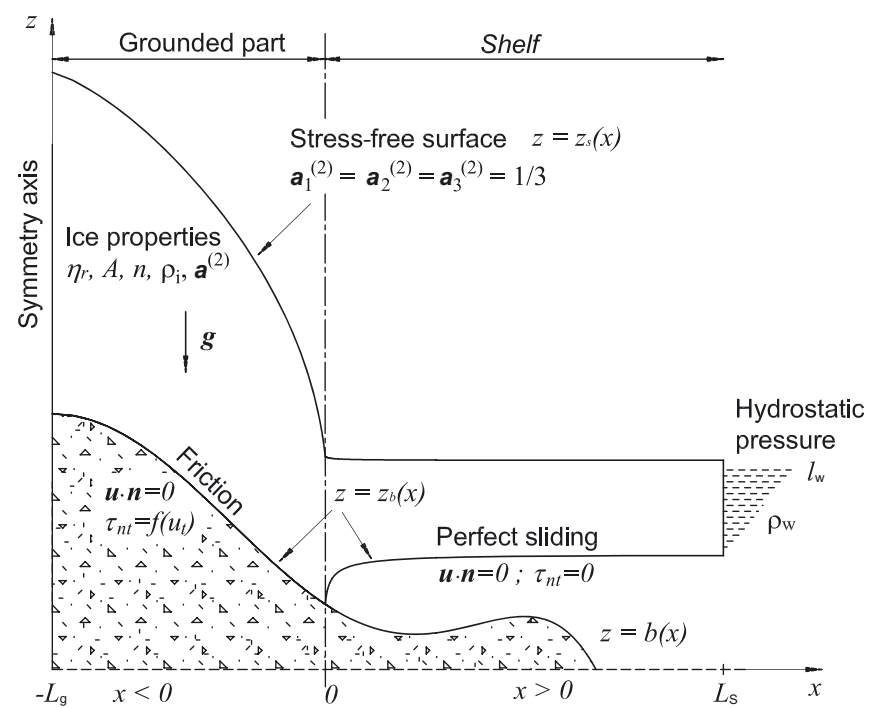

Fig. 1. Geometry of the ice sheet, notation and boundary conditions for the various experiments.

topography, in metres, is given as

$$
\begin{aligned}
b(x)=729 & -2184.8 \times\left[\frac{\left(x-L_{\mathrm{g}}\right)}{750 \mathrm{~km}}\right]^{2} \\
& +1031.72 \times\left[\frac{\left(x-L_{\mathrm{g}}\right)}{750 \mathrm{~km}}\right]^{4} \\
& -151.72 \times\left[\frac{\left(x-L_{\mathrm{g}}\right)}{750 \mathrm{~km}}\right]^{6},
\end{aligned}
$$

where $L_{g}$ is the length of the grounded part of the ice sheet. Using this bedrock and a uniform and constant surface accumulation of $0.3 \mathrm{ma}^{-1}$, the purpose of the MISMIP experiments was to compute various steady-state geometry configurations by varying Glen's flow-law parameter, $A$. In this paper, only diagnostic simulations are performed and the upper and lower free surface, $z=z_{\mathrm{s}}(x)$ and $z=z_{\mathrm{b}}(x)$, respectively, are fixed and equal to the steady solutions obtained at step 12 (i.e. for $A=7.89 \mathrm{MPa}^{-3} \mathrm{a}^{-1}$ ). The grounding-line position is also fixed and is arbitrarily taken to be $x=0$. Diagnostic simulations are justified since the objective here is to infer the velocity enhancement due to ice anisotropy by comparing isotropic and anisotropic flows for the same geometry.

Ice-flow variables, velocity and isotropic pressure, are computed by solving the following field and boundary equations:

$$
\begin{aligned}
& \operatorname{div} \boldsymbol{u}=0, \\
& z_{\mathrm{b}} \leq z \leq z_{\mathrm{s}} \\
& \operatorname{div} \boldsymbol{\sigma}+\rho_{\mathrm{i}} \boldsymbol{g}=0 \text {, } \\
& z_{\mathrm{b}} \leq z \leq z_{\mathrm{s}} \\
& \boldsymbol{\sigma} \cdot \boldsymbol{n}=\mathbf{0}, \\
& z=z_{\mathrm{s}} \text {, } \\
& \left.\begin{array}{r}
\tau_{n t}=\left.\boldsymbol{t} \cdot(\boldsymbol{\sigma} \cdot \boldsymbol{n})\right|_{\mathrm{b}}=C u_{t}^{m} \\
\boldsymbol{u} \cdot \boldsymbol{n}=0
\end{array}\right\} \quad z=z_{\mathrm{b}} ; x \leq 0, \\
& \left.\begin{array}{r}
\left.\boldsymbol{t} \cdot(\boldsymbol{\sigma} \cdot \boldsymbol{n})\right|_{\mathrm{b}}=0 \\
\boldsymbol{u} \cdot \boldsymbol{n}=0
\end{array}\right\} \quad z=z_{\mathrm{b}} ; x>0, \\
& u=0, \quad x=-L_{g}, \\
& \sigma_{n n}=-\max \left[\rho_{\mathrm{w}} g\left(I_{\mathrm{w}}-z_{\mathrm{b}}\right), 0\right], x=L_{\mathrm{s}} .
\end{aligned}
$$

Equations (8) and (9) express mass and momentum conservation, respectively. Here $\boldsymbol{u}$ is the velocity vector, $\boldsymbol{\sigma}=\boldsymbol{\tau}-p \boldsymbol{I}$ is
Table 1. Values of the parameters used in this study

\begin{tabular}{lc}
\hline Parameter & Value \\
\hline$A$ & $2.5 \times 10^{-2} \mathrm{MPa} \mathrm{m}^{-1 / 3} \mathrm{a}^{1 / 3}$ \\
$C$ & $9.81 \mathrm{~m} \mathrm{~s}^{-2}$ \\
$g$ & 10 \\
$k_{\mathrm{s}}$ & 0.4 \\
$k_{\mathrm{c}}$ & $732 \mathrm{~km}$ \\
$L_{\mathrm{g}}$ & $1068 \mathrm{~km}$ \\
$L_{\mathrm{s}}$ & $1 / 3$ \\
$m$ & 3 \\
$n$ & 0.06 \\
$\alpha$ & $1000 \mathrm{~kg} \mathrm{~m}^{-3}$ \\
$\rho_{\mathrm{w}}$ & $900 \mathrm{~kg} \mathrm{~m}^{-3}$ \\
$\rho_{\mathrm{i}}$ & \\
\hline
\end{tabular}

the Cauchy stress tensor, $p$ is the isotropic pressure, $\rho_{\mathrm{i}}$ and $\rho_{\mathrm{W}}$ are the density of ice and sea water, respectively, $I_{w}$ is the sea level, $\boldsymbol{n}$ and $\boldsymbol{t}$ are the normal and tangential unit vectors to the free surfaces and $\boldsymbol{g}=(0,0,-g)$ is the gravity vector. The upper surface is stress-free (Equation (10)) and for the lower surface the boundary conditions depend on whether the ice is grounded or floating. Where ice is grounded, the normal velocity is null and a Weertman-type friction law applies (Equation (11)), with $C$ the friction parameter, whereas for the ice shelf bottom both normal velocity and tangential friction are null (Equation (12)). The left boundary condition (Equation (13)) expresses a symmetry axis in $x=-L_{g}$, and the right boundary condition (Equation (14)) is the end of the ice-shelf front, $x=L_{s}$. In the case of evolving fabric, the Stokes equations are coupled using the constitutive relation (Equation (4)) to the orientation tensor evolution equations (Equation (5)), as explained by Gillet-Chaulet and others (2006). The values of the various parameters in the equations are listed in Table 1. This set of equations is solved using the finite-element code Elmer/lce; details on the numerics are given by Gillet-Chaulet and others (2006).

\section{NUMERICAL EXPERIMENTS \\ Definition of the enhancement factors}

The objective of these simulations is to infer the value of the enhancement factors for the grounded part, $E_{S I A}$, and the ice-shelf part, $E_{\mathrm{SSA}}$, by comparing anisotropic and isotropic results. It is easy to define, for each depth, values of the enhancement factors as a function of the local anisotropy and a given state of stress, but the objective here is to quantify the vertically integrated effect of anisotropy. Indeed, some models are vertically integrated, and the enhancement factors must then be determined from the global anisotropy effect from the surface to the bedrock.

For the grounded part, the dominant stress is the horizontal shear stress. From our full-Stokes anisotropic model, the enhancement in shear should be computed by comparing the shear strain rate, $\dot{\varepsilon}_{x y}$, for the anisotropic and isotropic cases. Because the shear strain rate varies with depth, the comparison has to be done on the resulting horizontal velocity, which is the horizontal surface velocity, $u_{s}$, minus the basal velocity, $u_{\mathrm{b}}$ (where basal sliding exists). Therefore, the enhancement factor in shear is determined as

$$
E_{\mathrm{SIA}}=\frac{u_{\mathrm{s}}^{\text {aniso }}-u_{\mathrm{b}}^{\text {aniso }}}{u_{\mathrm{s}}^{\text {iso }}-u_{\mathrm{b}}^{\text {iso }}} .
$$


Table 2. Settings for the various simulations. See text for explanations

\begin{tabular}{lccc}
\hline Test & Domain & Fabric & Sliding \\
\hline Exp. 1 & Sheet and shelf & SMAX & No \\
Exp. 2 & Sheet and shelf & SMAX & Yes \\
Exp. 3 & Shelf & Girdle & - \\
Exp. 4 & Shelf & Evolved & - \\
\hline
\end{tabular}

The enhancement factor, $E_{S I A}$, is thus only evaluated from the part of the horizontal velocity induced by shear deformation. With our definition of the polycrystal anisotropy, we know that $E_{\mathrm{SIA}} \leq k_{\mathrm{s}}$, equality being reached in the particular case of a homogeneous single-maximum fabric over all the domain.

For the ice shelf, the dominant stress is horizontal tension. The enhancement factor for the ice shelf must then be computed by comparing the horizontal strain rate, $\dot{\varepsilon}_{x x}$, for anisotropic and isotropic cases. In the case of perfect basal sliding, $\dot{\varepsilon}_{x x}$ is almost constant with depth, so the enhancement factor can be evaluated at any elevation in the ice shelf. However, the SSA is also used to determine the icestream basal velocity. In this case, the tension enhancement factor should be inferred from the bottom value of the horizontal strain rate, $\left.\dot{\varepsilon}_{x x}\right|_{\mathrm{b}}$. Therefore, we define the tension enhancement factor by

$$
E_{S S A}=\frac{\left.\dot{\varepsilon}_{X X}^{\text {aniso }}\right|_{b}}{\left.\dot{\varepsilon}_{X X}^{\text {iso }}\right|_{b}} .
$$

From the definition of the polycrystal anisotropy, the tension enhancement factor, $E_{S S A}$, should be greater than the anisotropy factor in compression, $k_{\mathrm{C}}\left(E_{\mathrm{SSA}} \geq k_{\mathrm{C}}\right)$. Here again, the equality is reached for a homogeneous single-maximum fabric over the whole ice shelf.

\section{Enhancement factor for grounded ice}

The experiments are listed in Table 2. For all anisotropic experiments, the corresponding isotropic experiment (not listed) is used as the reference to compute the enhancement factor (Equations (15) and (16)). For the grounded part, two experiments were performed for various basal conditions, one assuming no sliding at the base (exp. 1) and the second with basal sliding (exp. 2). For these two experiments, the fabric is given and its evolution is not computed. Due to the fixed geometry and the strong changes in the flow condition at the grounding line, it was not possible to achieve a steadystate fabric field. Nevertheless, as shown below, the fabric was computed on the ice shelf where we did not have data input, using a simplified geometry model.

As shown in Figure 2 (dashed curve), for these two experiments the applied (fixed) fabric is a single-maximum fabric which becomes more and more concentrated from the surface to the bedrock as a function of the reduced depth. This is a typical fabric profile under a dome where ice is submitted to a compression stress, but it applies also along a flank where, due to shear, the fabric is known to evolve to a single-maximum pattern near the bedrock (Seddik and others, 2008). In what follows, this imposed fabric profile is referred to as the single-maximum (SMAX) fabric profile.

Results for the grounded part are shown in Figure 3. As expected (Mangeney and Califano, 1998; Gagliardini and Meyssonnier, 1999; Staroszczyk and Morland, 2000), the surface velocity, $u_{s}$, for the anisotropic fabric is greater than

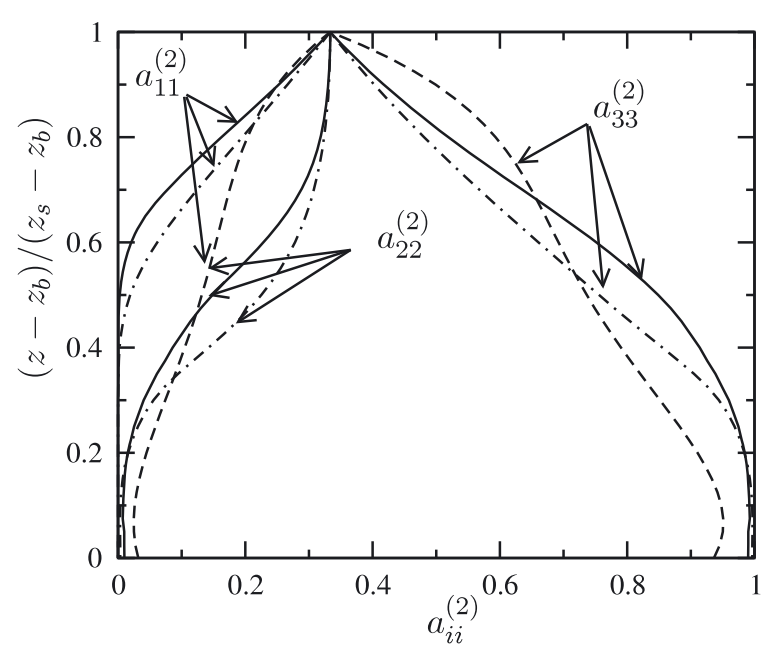

Fig. 2. Vertical fabric profiles given as the evolution of the diagonal components of the second-order orientation tensor, $\mathbf{a}^{(2)}$, for the imposed single-maximum (SMAX) fabric (dashed curve) and the computed steady-state solution in the ice shelf at $x=320 \mathrm{~km}$ (solid curve) and $x=800 \mathrm{~km}$ (dotted curve).

that of the isotropic fabric, which confirms that the anisotropy makes the ice softer in the grounded part, where shear stress dominates. The enhancement factor evolution along $x$ is calculated using Equation (15) for both with basal sliding and no basal sliding. Even if the surface velocities in the case of basal sliding are much greater than those in the case of no sliding, for the same anisotropy, both give the same enhancement factor $E_{S I A}=4.5$. The same values of $E_{S I A}$ derived from two experiments with various basal conditions indicate that, here, basal sliding has almost no effect on the ice shear deformation. It should be noted that the enhancement factor is also uniform on all the grounded part. Mangeney and others (1996) obtained an enhancement factor of 1.7 and Gagliardini and Meyssonnier (2002) found a value of 1.75 . These small values are explained by the uniform-stress polycrystal model used in their approach, which had a limited anisotropy factor, $k_{\mathrm{s}}<2.5$. With $k_{\mathrm{s}}=5$, Staroszczyk and Morland (2000) obtained an enhancement factor of 4 . We recall that in our work $K_{s}$ is set to its experimental value of 10.1 .

Certainly because the simulations are performed using a fixed geometry, irregularities of the velocity field are observed in the vicinity of the grounding line (over a distance of a few ice thicknesses). Because the problem of the grounding-line dynamics is not the intended subject of this paper, results in the vicinity of the grounding line are not discussed. These irregularities of the velocity field also explain why it was not possible to compute the steadystate fabric field associated with this ice-sheet geometry. At the dome, because of the vanishing horizontal velocity, the enhancement factor, $E_{S I A}$, is not defined.

\section{Enhancement factor for the ice shelf}

As the ice shelf is dominated by longitudinal stretching and lateral shearing instead of horizontal shearing, application of the SMAX fabric profile is inappropriate. We performed two furher experiments, exp. 3 and exp. 4, to infer the influence of fabric type on the flow. For these experiments, the domain was restricted to the ice-shelf part in order to be able to compute the steady-state fabric field, as depicted in 


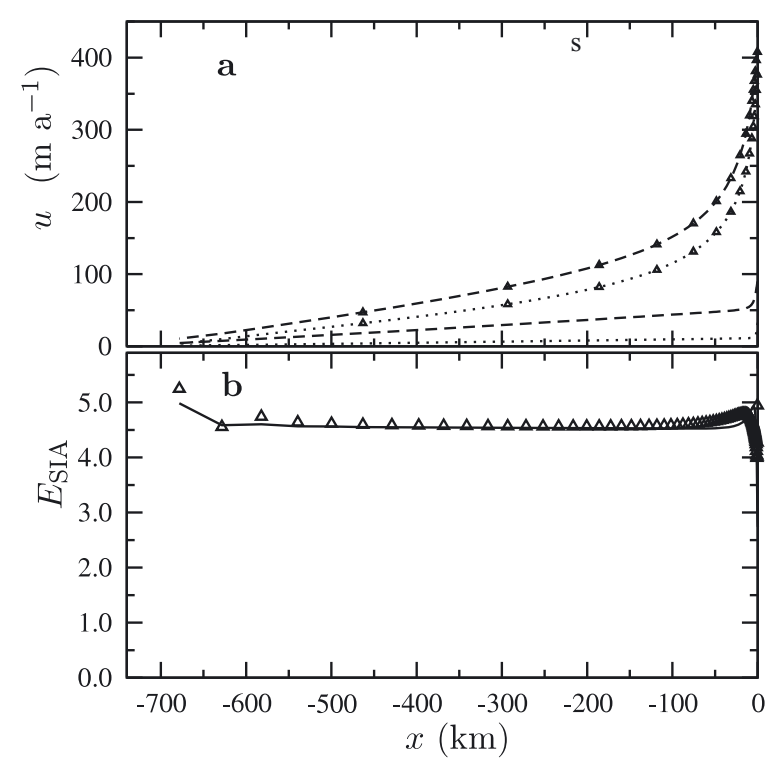

Fig. 3. (a) Horizontal velocity at the surface of the grounded part of the ice sheet as a function of distance from the grounding line, for the isotropic fabric (dotted curves) and SMAX fabric profile (dashed curves), and with and without sliding (triangle and nosymbol curves, respectively). (b) Corresponding enhancement factor in shear evaluated from Equation (15) in the case of sliding (triangles) and no-sliding (no symbol).

Figure 4. For this restricted geometry, all the flow equations are the same, except that the left-side boundary conditions (Equation (13)) are modified to impose an inflow flux given by $u(0, z)=50 \mathrm{~m} \mathrm{a}^{-1}$ and $v(0, z)=0$. This imposed inflow velocity corresponds approximately to the mean horizontal velocity at the grounding line for exp. 1 .

In exp. 3 a constant girdle fabric $\left(a_{1}^{(2)}=0.01, a_{2}^{(2)}=a_{3}^{(2)}=\right.$ 0.495), representing the expected fabric for a longitudinal uniaxial tension stress, is imposed on all of the ice-shelf domain. For exp. 4 the fabric field is computed as the steadystate solution of the coupling between the flow equations and the fabric-evolution equations, assuming a SMAX profile as a boundary condition for the inflow boundary, $x=0$. We checked that, whatever the initial fabric in the ice shelf (isotropic or SMAX profile), the problem converges to the same steady solution. This test with various initial conditions allows us to check whether the steady state of the ice flow is

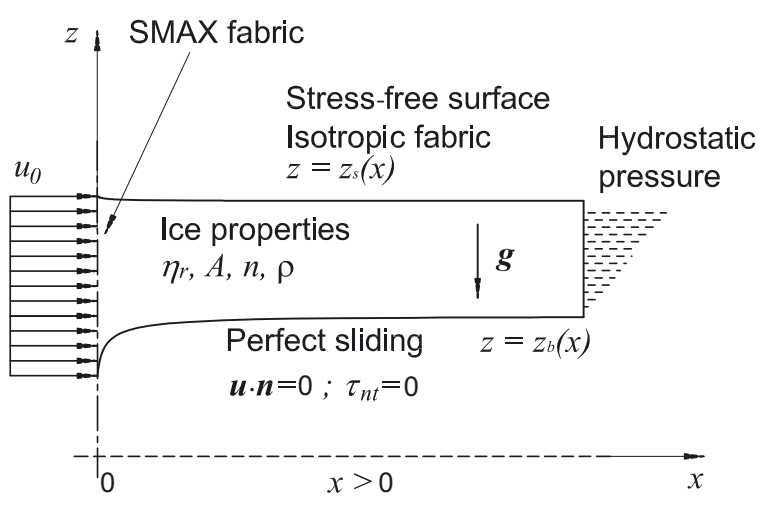

Fig. 4. Geometry and boundary conditions for the ice-shelf experiments.

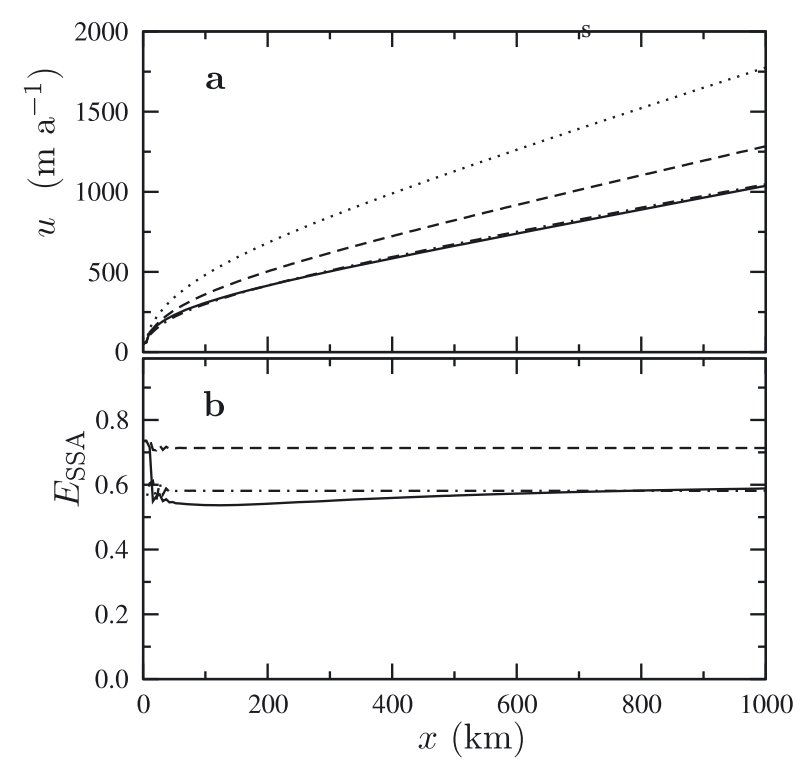

Fig. 5. (a) Horizontal velocity at the surface of the ice shelf as a function of the distance from the grounding line, for the isotropic fabric (dotted curve), SMAX fabric profile (dashed curve), girdle fabric (dot-dashed curve) and computed steady-state fabric (solid curve). (b) Corresponding enhancement factor in tension evaluated from Equation (16).

achieved or not and validates the numerical method used to compute the fabric field. The steady-state fabric profiles for the ice shelf at $x=320$ and $800 \mathrm{~km}$ are shown in Figure 2 (solid and dotted curves, respectively). The evolution with depth of the computed fabric is different from the SMAX one but, due to the plane strain hypothesis, the fabric does not evolve to a girdle fabric. Comparison of these fabric profiles taken at two different locations indicates that the vertical evolution of the fabric is fairly similar throughout the ice shelf.

Results for the ice-shelf part are presented in Figure 5. In the absence of horizontal shearing, the velocity is constant with depth and its value increases almost linearly with distance to the grounding line. The surface velocities for the isotropic case and the three anisotropic cases (SMAX, girdle and steady-state fabrics) are shown in Figure 5a. The surface velocity, $u_{\mathrm{s}}$, for the isotropic case is greater than any one of the anisotropic cases, indicating that anisotropic ice is stiffer under the stress conditions prevailing in ice shelves. For each experiment, the corresponding enhancementfactor evolution along the flow direction, inferred from Equation (16), is shown in Figure 5b. As for the grounded part, for a given experiment, the value of the enhancement factor, $E_{S S A}$, is almost uniform over the whole ice shelf. Its value is 0.71 for the SMAX fabric and 0.58 for the girdle fabric. For the computed steady-state fabric, the mean value of $E_{\mathrm{SSA}}$ is $\sim 0.57$.

We also tested imposing an isotropic fabric as a boundary condition for the inflow boundary $x=0$ (instead of a SMAX profile) and varying the value of the inflow velocity (from $u(0, z)=50$ up to $300 \mathrm{~m} \mathrm{a}^{-1}$ ). All the results (not shown) indicate that the inferred enhancement factors, $E_{S S A}$, for these cases range between 0.52 and 0.71 , greater values being obtained for the SMAX fabric profile. For the flow regime prevailing in ice shelves, whatever the fabrics, the flow enhancements induced by ice anisotropy are very similar. 


\section{Effect of non-isothermal conditions}

In these applications, enhancement factors were inferred using isothermal conditions, whereas ice-sheet models use a non-constant temperature field. For the grounded part, increasing temperature with depth leads to a greater value of the enhancement factor, $E_{S I A}$, because it increases the shear deformation near the bedrock where the fabric is the most concentrated. We performed simulations assuming a fixed temperature field function of the reduced depth as observed at the Greenland Icecore Project (GRIP) site (fig. 2a in Gagliardini and Meyssonnier, 2000). These results indicate that for a variable temperature the enhancement factor, $E_{S I A}$, is equal to 5.6. Finally, isothermal and non-isothermal simulations give a range for $E_{S I A}$ of 4.5-5.6, the greater value obtained for a non-constant temperature certainly being the more realistic.

For the ice shelf, the steady-state fabric obtained with isothermal conditions was used to test the influence of variable temperature conditions, assuming the same temperature profile as for the grounded part. The value of the enhancement factor in tension for a variable temperature is $E_{\mathrm{SSA}}=0.60$, to be compared with the value of 0.57 obtained with the isothermal conditions. These results indicate that an evolving temperature with depth has almost no influence on the resulting enhancement factor. In tension, contrary to what happens in shear, the flow is controlled by the layer with the lesser fluidity, i.e. the upper layers where the temperature is low.

\section{Which enhancement factor for ice streams?}

In the previous subsections, we have shown that the calculated enhancement factors in shear, $E_{S I A}$, and in tension, $E_{\mathrm{SSA}}$, are almost uniform over the grounded part and the iceshelf part, respectively. Let us now consider the transition zone between these two parts, where both the longitudinal and shear stress have the same magnitude. For this purpose, exp. 2 (SMAX fabric profile and sliding) was repeated for different friction coefficients, ranging from $0.3 C$ to $1.2 C$, where $C$ is the value used in the previous simulations and is given in Table 1. As shown in Figure 6, the value of the enhancement factor in tension, $E_{\mathrm{SSA}}$, decreases from 1, where sliding is negligible, to its ice-shelf value $\left(E_{S S A}=0.71\right)$ just downstream of the grounding line. The smaller the friction parameter is, the longer the stream, and the decrease of $E_{\mathrm{SSA}}$ follows the stream length. This variation of $E_{\mathrm{SSA}}$ characterizes the fact that the longitudinal stress becomes progressively greater than the shear stress as the ice moves down in the ice stream.

Therefore, the value of the enhancement factor used in the SSA, if it is being applied to an ice stream, should not be constant and equal to that of the ice shelf, but should decrease from 1 at the onset of the ice stream to its ice-shelf value at the grounding line.

Again, because of the fixed domain geometry, the diagnostic velocity field in the vicinity of the grounding line is not smooth, inducing large oscillations of the enhancement factor in tension.

\section{CONCLUSIONS}

In this work, we have estimated the enhancement factors induced solely by the ice anisotropy for an ice sheet with a grounded part and an ice shelf. For this purpose, anisotropic

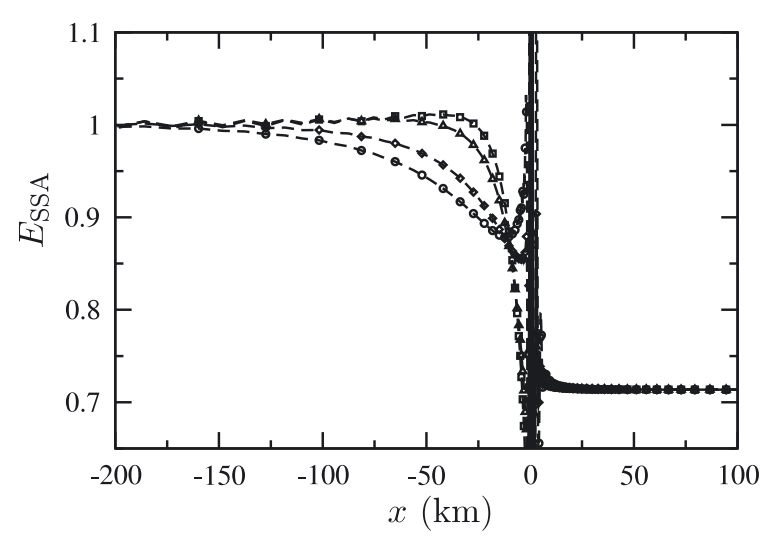

Fig. 6. Enhancement factor in tension, $E_{S S A}$, as a function of distance to grounding line, evaluated from Equation (16) for different friction parameters from faster flow, $0.3 C$ (circles) and $0.5 C$ (lozenges), to slower flow, $C$ (triangles) and $1.2 C$ (squares). The value of $C$ is given in Table 1 and corresponds to that used in the previous experiments.

and isotropic models were applied simultaneously and compared. For grounded ice the anisotropic flow is computed assuming a fixed fabric profile, as observed below a dome. Two basal boundary conditions (no sliding and sliding) were applied. The results show that the basal sliding has almost no effect on the enhancement factor for the grounded part. For the isothermal case, the value of the enhancement factor is 4.5 , whereas it is 5.6 in the regime where temperature increases with depth.

For the floating part, owing to the lack of knowledge of the distribution of the fabric with depth, we conducted simulations in which the fabric was evolved to obtain the steady-state fabric field. A single-maximum fabric profile and a constant girdle fabric were also imposed on the ice shelf. These applications with different fabric fields show that the value of the enhancement factor in tension ranges from 0.58 to 0.71 .

These two different values of the enhancement factor for the grounded ice and the ice shelf can be quantitatively explained by the anisotropic behaviour of polar ice and the difference of stress regime between the grounded and floating parts. In a large-scale model, a contrast ratio $E_{\mathrm{SIA}} / E_{\mathrm{SSA}}$ ranging between 5 and 10 should be used to empirically account for the anisotropy effect in the various parts of the ice sheet.

Many other phenomena, such as basal sliding for grounded ice or basal melting below ice shelves, are also not well constrained in ice-sheet models, so the enhancement factors used by ice-sheet modellers not only contain an adjustment for ice anisotropy but, possibly, also for these poorly known bottom boundary conditions. In fact, some of the anisotropy effects might be accounted for by adjusting basal conditions instead of ice rheology, as demonstrated by Bueler using an inverse method constrained by the surface velocity of Greenland (personal communication from E. Bueler, 2010).

It should be noted that anisotropy induces other complex effects that cannot be properly modelled using an isotropic law and the concept of an enhancement factor. The use of enhancement factors should therefore be restricted to large-scale ice-sheet models in which the simplified Stokes equations do not allow an anisotropic flow law to be properly incorporated. For higher-order or full-Stokes models, a 
more accurate way of dealing with anisotropy requires the implementation of an anisotropic flow law.

\section{ACKNOWLEDGEMENTS}

This research is a part of the NEEM (North Eemian) project funded by the Agence National de la Recherche (ANR), France, and the ice2sea project. Y. Ma was funded by the ANR NEEM project. The ice2sea project is funded by the European Commission's 7th Framework Programme through grant No. 226375 (ice2sea manuscript No. 005). We appreciate helpful comments from E. Bueler, an anonymous reviewer and the scientific editor, R. Greve, who considerably improved the final quality of the paper.

\section{REFERENCES}

Bueler, E. and J. Brown. 2009. Shallow shelf approximation as a 'sliding law' in a thermomechanically coupled ice sheet model. J. Geophys. Res., 114(F3), F03008. (10.1029/2008JF001179.)

Castelnau, O., P. Duval, R. Lebensohn and G.R. Canova. 1996. Viscoplastic modeling of texture development in polycrystalline ice with a self-consistent approach: comparison with bound estimates. J. Geophys. Res., 101(B6), 13,851-13,868.

Castelnau, O. and 7 others. 1998. Anisotropic behavior of GRIP ices and flow in central Greenland. Earth Planet. Sci. Lett., 154(1-4), 307-322.

Craven, M. and 7 others. 2005. Borehole imagery of meteoric and marine ice layers in the Amery Ice Shelf, East Antarctica. J. Glaciol., 51(172), 75-84.

Craven, M., I. Allison, H.A. Fricker and R. Warner. 2009. Properties of a marine ice layer under the Amery Ice Shelf, East Antarctica. J. Glaciol., 55(192), 717-728.

Durand, G. and 9 others. 2007. Change of the ice rheology with climatic transitions. Implication on ice flow modelling and dating of the EPICA Dome C core. Clim. Past, 3, 155-167.

Durand, G. and 7 others. 2009a. Evolution of the texture along the EPICA Dome C ice core. In Hondoh, T., ed. Physics of ice core records II. Hokkaido, Hokkaido University. Institute of Low Temperature Science, 91-105..

Durand, G., O. Gagliardini, B. de Fleurian, T. Zwinger and E. Le Meur. 2009b. Marine ice-sheet dynamics: hysteresis and neutral equilibrium. J. Geophys. Res., 114(F3), F03009. (10.1029/2008JF001170.)

Eisen, O., I. Hamann, S. Kipfstuhl, D. Steinhage and F. Wilhelms. 2007. Direct evidence for continuous radar reflector originating from changes in crystal-orientation fabric. Cryosphere, 1(1), $1-10$.

Fricker, H.A., S. Popov, I. Allison and N. Young. 2001. Distribution of marine ice under the Amery Ice Shelf, East Antarctica. Geophys. Res. Lett., 28(11), 2241-2244.

Gagliardini, O. and J. Meyssonnier. 1999. Plane flow of an ice sheet exhibiting strain-induced anisotropy. In Hutter, K., Y. Wang and $\mathrm{H}$. Beer, eds. Advances in cold-region thermal engineering and sciences: technological, environmental, and climatological impact. Berlin, etc., Springer-Verlag, 171-182.

Gagliardini, O. and J. Meyssonnier. 2000. Simulation of anisotropic ice flow and fabric evolution along the GRIP-GISP2 flowline, central Greenland. Ann. Glaciol., 30, 217-223.
Gagliardini, O. and J. Meyssonnier. 2002. Lateral boundary conditions for a local anisotropic ice-flow model. Ann. Glaciol., 35, 503-509.

Gillet-Chaulet, F., O. Gagliardini, J. Meyssonnier, M. Montagnat and O. Castelnau. 2005. A user-friendly anisotropic flow law for icesheet modelling. J. Glaciol., 51(172), 3-14.

Gillet-Chaulet, F., O. Gagliardini, J. Meyssonnier, T. Zwinger and J. Ruokolainen. 2006. Flow-induced anisotropy in polar ice and related ice-sheet flow modelling. J. Non-Newtonian Fluid Mech., 134(1-3), 33-43.

Hutter, K. 1983. Theoretical glaciology; material science of ice and the mechanics of glaciers and ice sheets. Dordrecht, etc., D. Reidel Publishing Co./Tokyo, Terra Scientific Publishing Co.

Huybrechts, P. 1990. A 3-D model for the Antarctic ice sheet: a sensitivity study on the glacial-interglacial contrast. Climate Dyn., 5(2), 79-92.

Kirchner, N. and S. Faria. 2009. The multiscale structure of Antarctica. Part II: ice shelves. In Hondoh, T., ed. Physics of ice core records II. Hokkaido, Hokkaido University. Institute of Low Temperature Science, 61-71.

MacAyeal, D.R. 1989. Large-scale ice flow over a viscous basal sediment: theory and application to Ice Stream B, Antarctica. J. Geophys. Res., 94(B4), 4071-4087.

Mangeney, A. and F. Califano. 1998. The shallow ice approximation for anisotropic ice: formulation and limits. J. Geophys. Res. 103(B1), 691-706.

Mangeney, A., F. Califano and O. Castelnau. 1996. Isothermal flow of an anisotropic ice sheet in the vicinity of an ice divide. J. Geophys. Res., 101(B12), 28,189-28,204.

Martín, C., R.C.A. Hindmarsh and F.J. Navarro. 2009. On the effects of divide migration, along-ridge flow, and basal sliding on isochrones near an ice divide. J. Geophys. Res., 114(F2), F02006. (10.1029/2008JF001025.)

Pettit, E.C., T. Thorsteinsson, H.P. Jacobson and E.D. Waddington. 2007. The role of crystal fabric in flow near an ice divide. J. Glaciol., 53(181), 277-288.

Philip, A. and J. Meyssonnier. 1999. Anisotropic isothermal ice-cap flow with the shallow ice approximation. In Hutter, K., Y. Wang and $\mathrm{H}$. Beer, eds. Advances in cold-region thermal engineering and sciences: technological, environmental, and climatological impact. Berlin, etc., Springer-Verlag, 237-248.

Pimienta, P., P. Duval and V.Y. Lipenkov. 1987. Mechanical behavior of anisotropic polar ice. IAHS Publ. 170 (Symposium at Vancouver 1987 - The Physical Basis of Ice Sheet Modelling), $57-66$.

Placidi, L., R. Greve, H. Seddik and S.H. Faria. 2010. Continuummechanical, anisotropic flow model for polar ice masses, based on an anisotropic flow enhancement factor. Contin. Mech. Thermodyn., 22(3), 221-237.

Seddik, H., R. Greve, L. Placidi, I. Hamann and O. Gagliardini. 2008. Application of a continuum-mechanical model for the flow of anisotropic polar ice to the EDML core, Antarctica. J. Glaciol., 54(187), 631-642.

Staroszczyk, R. and L.W. Morland. 2000. Plane ice-sheet flow with evolving orthotropic fabric. Ann. Glaciol., 30, 93-101.

Thorsteinsson, T., J. Kipfstuhl and H. Miller. 1997. Textures and fabrics in the GRIP ice core. J. Geophys. Res., 102(C12), $26,583-26,599$.

Treverrow, A. 2009. The flow of polycrystalline anisotropic ice: laboratory and model studies. (PhD thesis, University of Tasmania.)

Van der Veen, C.J. and I.M. Whillans. 1994. Development of fabric in ice. Cold Reg. Sci. Technol., 22(2), 171-195. 